\title{
PROPHET INEQUALITIES AND ORDER SELECTION IN OPTIMAL STOPPING PROBLEMS
}

\author{
T. P. HILL
}

\begin{abstract}
A complete determination is made of the possible values for $E\left(\sup X_{n}\right)$ and $\sup \left\{E X_{t}: t\right.$ a stop rule $\}$ for $X_{1}, X_{2}, \ldots$ independent uniformly bounded random variables; this yields results of Krengel, Sucheston, and Garling, and of Hill and Kertz as easy corollaries.

In optimal stopping problems with independent random variables where the player is free to choose the order of observation of these variables it is shown that the player may do just as well with a prespecified fixed ordering as he can with order selections which depend sequentially on past outcomes.

A player's optimal expected gain if he is free to choose the order of observation is compared to that if he is not; for example, if the random variables are nonnegative and independent, he may never do better than double his optimal expected gain by rearranging the order of observation of a given sequence.
\end{abstract}

1. Introduction. Following the initial discoveries by Krengel and Sucheston [7] of surprising universal constants in the theory of optimal stopping, further comparisons have been made $[1,3,4,5,8]$ between $E\left(\sup X_{n}\right)$, "the expected gain of a prophet or player with complete foresight", and $\sup \left\{E X_{t}: t\right.$ a stop rule $\}$, "the expected gain of a player using nonanticipating stop rules".

In $\S 2$, a complete determination is made (Theorem 2.3) of the possible values for $\sup E X_{t}$ and $E\left(\sup X_{n}\right)$ in the case of uniformly bounded independent random variables.

In $\S 3$ it is shown (Theorem 3.11) that in an optimal stopping problem (with independent random variables) in which the player is free to select the order of observation of the variables, he may do just as well with a prespecified fixed ordering as with order selections depending on past outcomes.

In $\$ 4$ a complete determination is made (Theorem 4.1) of the advantage a player may obtain by rearranging, even as a function of past outcomes, the order of observation of a given sequence.

2. Prophet inequalities. Throughout this paper, $X_{1}, X_{2}, \ldots$ will be a sequence of independent random variables on a probability space $(\Omega, \mathbb{Q}, P), \mathbf{N}$ and $\mathbf{R}$ will denote the natural and real numbers, respectively, $\mathbf{B}$ and $\mathbf{B}^{\infty}$ will denote the Borel sigma fields on $\mathbf{R}$ and $\mathbf{R}^{\infty}$, and $E(X \mid Y)$ the conditional expectation of $X$ given $Y$.

Received by the editors March 8, 1982 and, in revised form, September 24, 1982. Presented at the Annual AMS meeting in Cincinatti in January 82.

1980 Mathematics Subject Classification. Primary 60G40.

Key words and phrases. Optimal stopping theory, prophet inequalities 
Definition 2.1. A function $t: \mathbf{R}^{\infty} \rightarrow \mathbf{N}$ is a (measurable) stopping function if $t^{-1}(n) \in \mathbf{B}^{\infty}$ for all $n$, and if $t\left(x_{1}^{\prime}, x_{2}^{\prime}, \ldots\right)=n$ whenever $t\left(x_{1}, x_{2}, \ldots\right)=n$ and $x_{i}^{\prime}=x_{i}$ for all $i=1,2, \ldots, n$. $\mathcal{T}$ is the set of all stopping functions, and $\mathcal{T}_{n}$ the set of all stopping functions which stop no later than $n$, i.e. $\mathcal{T}_{n}=\{t \in \mathcal{T}: t \leqslant n$ everywhere $\}$. For $t \in \mathcal{T}, X_{t}: \Omega \rightarrow \mathbf{R}$ is the function defined by $X_{t}(\omega)=X_{n}(\omega)$ for all $\omega$ with $t\left(X_{1}(\omega), X_{2}(\omega), \ldots\right)=n$.

For the remainder of this section, one may think of $\mathcal{T}_{\text {and }} \mathcal{T}_{n}$ as the classically defined sets of stop rules for $\left(X_{1}, X_{2}, \ldots\right)$ and $\left(X_{1}, \ldots, X_{n}\right)$ respectively; in all subsequent results in this section the definitions coincide. The main reason for the approach of Definition 2.1 is that $\$ \S 3$ and 4 will consider situations where a player is allowed to select the order of observations of the $\left\{X_{i}\right\}$, and stop whenever he pleases, whereas the classical definition of stop rule is dependent on one given ordering of the random variables. Moreover, as remarked in [6], this present approach seems more natural in the sense that actual implementation of stop rules invariably involves only sets of real values with which the player is content to stop, not observation of the underlying subsets of $\Omega$.

Definition 2.2. $v=V\left(X_{1}, X_{2}, \ldots\right)=\sup \left\{E X_{t}: t \in \mathcal{T}\right\}$, and $u=E\left(\sup _{n} X_{n}\right)$.

The main result in this section is

THEOREM 2.3. The set of all possible values of the ordered pairs $(v, u)$ for independent random variables uniformly bounded in $[0,1]$ is precisely the closed convex set $S$ in $\mathbf{R}^{2}$ given by $S=\left\{(x, y): x \leqslant y \leqslant 2 x-x^{2} ; 0 \leqslant x \leqslant 1\right\}$.

Proof. To see that every point in $S$ is a possible value $(v, u$ ) (for some $\left.X_{1}, X_{2}, \ldots\right)$, fix $(x, y)$ in $S$, let $\alpha=(y-x) /\left(x-x^{2}\right)$, and define $X_{1}, X_{2}, \ldots$ by: $X_{1} \equiv x ; X_{2}=1$ with probability $\alpha x$, and $=0$ otherwise; and $X_{n} \equiv 0$ for $n>2$. Then it is easy to check that $v=\sup \left\{E X_{t}: t \in \mathcal{T}\right\}=x$ (since $0 \leqslant \alpha \leqslant 1$ ), and that $u=E\left(\sup X_{n}\right)=\alpha x+x-\alpha x^{2}=y$.

Nearly all the work for the converse is found in the proof of Theorem A in [4]. Fix $X_{1}, X_{2}, \ldots$ independent and uniformly bounded in [0,1], and check (as in [4]) that $u-v=E\left(\sup _{n} X_{n}\right)-\sup \left\{E X_{t}: t \in \mathcal{T}\right\} \leqslant E\left(\max \left\{Y_{1}, Y_{2}\right\}\right)-\sup \left\{E Y_{t}: t \in \mathcal{T}_{2}\right\}$, where: $Y_{1} \equiv v$; and $Y_{2}=1$ with probability $v$, and $=0$ otherwise. Since

$$
\sup \left\{E Y_{t}: t \in \mathcal{T}_{2}\right\}=v \text { and } E\left(\max \left\{Y_{1}, Y_{2}\right\}\right)=2 v-v^{2} \text {, }
$$

this implies $u \leqslant 2 v-v^{2}$; the inequality $v \leqslant u$ is immediate from the definitions.

Corollary 2.4 (Krengel-Sucheston-Garling [8]). If $X_{1}, X_{2}, \ldots$ are independent and nonnegative, then

$$
E\left(\sup _{n} X_{n}\right) \leqslant 2 \sup \left\{E X_{t}: t \in \mathcal{T}\right\}
$$

Proof. Assume both sides of (1) are finite (otherwise both are infinite, and (1) is trivial), and by routine truncation and normalizing reduce to the case where the random variables are uniformly bounded in $[0,1]$. Apply the theorem. 
Corollary 2.5 (Hill-Kertz [4]). If $X_{1}, X_{2}, \ldots$ are independent and uniformly bounded in $[a, a+b]$, then

$$
E\left(\sup X_{n}\right)-\sup \left\{E X_{t}: t \in \mathcal{T}\right\} \leqslant b / 4 \text {. }
$$

Proof. Again reduce first to the case where the random variables are bounded in $[0,1]$. By the theorem, $u-v \leqslant v-v^{2}$, which in turn is $\leqslant \frac{1}{4}$ since $0 \leqslant v \leqslant 1$.

For the case where $X_{1}, X_{2}, \ldots$ are uniformly bounded in [a,a+b], it is easy to see that the set $S$ in Theorem 2.3 must be replaced by

$$
S^{\prime}=\left\{(x, y): x \leqslant y \leqslant 2(x-a)-(x-a)^{2} / b+a ; a \leqslant x \leqslant a+b\right\} .
$$

3. Order selection in optimal stopping problems. $\mathscr{P}_{n}$ will denote the set of all permutations of $\{1,2, \ldots, n\}$ and $\mathscr{P}$ the permutations of $\mathbf{N}$. For $\pi \in \mathscr{P}, \pi[j]$ will denote the image of $j$ under the mapping $\pi: \mathbf{N} \rightarrow \mathbf{N}$.

Definition 3.1. An order selector is a function $r: \mathbf{R}^{\infty} \rightarrow \mathcal{\rho}$ such that $r\left(x_{1}, x_{2}, \ldots\right)[1]$ is constant for all $\left(x_{1}, x_{2}, \ldots\right) \in \mathbf{R}^{\infty}$, and such that $r\left(x_{1}, x_{2}, \ldots\right)[j]$ is a Borel-measurable function of $x_{1}, \ldots, x_{j-1}$. That is, $r[j]^{-1}(k) \in \mathbf{B}^{\infty}$ for all $j, k \in \mathbf{N}$, and if $r\left(x_{1}, x_{2}, \ldots\right)[j]=k$, and $x_{i}^{\prime}=x_{i}$ for $i=1,2, \ldots, j-1$, then $r\left(x_{1}^{\prime}, x_{2}^{\prime}, \ldots\right)[j]=k$. (In stop rule terminology, $r[j]$ is "determined by" time $j-1$.) $\Re$ will denote the set of all order selectors, and $\mathcal{R}_{n}$ the set of all order selectors $r$ for which $r[j] \equiv j$ for $j>n$.

Without ambiguity, $\mathscr{P}$ and $\mathscr{P}_{n}$ will be viewed as the subsets of $\mathcal{R}$ and $\Re_{n}$ representing constant order selectors. Then it is easy to see from the definitions that $\mathscr{P}_{2}=\Re_{2}$, but that $\mathscr{P}_{n}$ is a proper subset of $\Re_{n}$ for all $n>2$.

Next is an example of a simple nonconstant order selector.

EXAMPLE 3.2. Define $\hat{r} \in \Re_{3}$ by: $\hat{r}[1] \equiv 2 ; \hat{r}\left(x_{1}, x_{2}, \ldots\right)[2]=1$ if $x_{1}>0$, and $=3$ otherwise; $\hat{r}\left(x_{1}, x_{2}, \ldots\right)[3]=3$ if $x_{1}>0$, and $=1$ otherwise; and $\hat{r}[j] \equiv j$ for $j>3$.

Definition 3.3. For $r \in \mathcal{R}$ and $t \in \mathcal{T}, X_{r[i]}$ is the random variable defined by $X_{r[t]}(\omega)=X_{n}(\omega)$ on the set where

$$
n=r\left(X_{r[1]}(\omega), X_{r[2]}(\omega), \ldots\right)\left[t\left(X_{r[1]}(\omega), X_{r[2]}(\omega), \ldots\right)\right] \text {. }
$$

The value of the order selector $r$ for the sequence $\left(X_{1}, X_{2}, \ldots\right), V\left(X_{r}\right)$, is given by $V\left(X_{r}\right)=\sup \left\{E\left(X_{r[t]}\right): t \in \mathcal{T}\right\}$.

(It should be noted that $V\left(X_{\pi}\right)=V\left(X_{\pi[1]}, X_{\pi[2]}, \ldots\right), V$ as in Definition 2.2, for all $\pi \in \mathcal{P}$.)

EXAMPLE 3.4. For $\hat{r}$ as in Example 3.2, the $\left\{X_{\hat{r}[j]}\right\}, j=1,2, \ldots$, are given by: $X_{\hat{r}[1]} \equiv X_{2} ; X_{\hat{r}[2]}=X_{1}$ if $X_{2}>0$, and $=X_{3}$ otherwise; $X_{\hat{r}[3]}=X_{3}$ if $X_{2}>0$, and $=X_{1}$ otherwise; and $X_{\hat{r}[j]}=X_{j}$ for $j>3$. Thus for fixed $t \in \mathcal{T}, E X_{\hat{r}[t]}=\int_{T_{1}} X_{2}+$ $\int_{T_{2} \cap D} X_{1}+\int_{T_{2} \cap D^{c}} X_{3}+\cdots$, where $T_{i}=\left\{\omega: t\left(X_{2}(\omega), X_{\hat{r}[2]}(\omega), \ldots\right)=i\right\}, i=1,2$, and $D=\left\{X_{2}>0\right\}$.

The following lemma, a consequence of backward induction, is stated here for ease of reference.

LeMma 3.5 [2, p. 50]. For $\pi \in \mathcal{P}$,

$$
V\left(X_{\pi}\right)=\int_{B} X_{\pi[1]}+P\left(B^{c}\right) \cdot V\left(X_{\pi[2]}, X_{\pi[3]}, \ldots\right),
$$

where $B=\left\{X_{\pi[1]}>V\left(X_{\pi[2]}, X_{\pi[3]}, \ldots\right)\right\}$. 
Definition 3.6. For $x \in \mathbf{R}$ and $r \in \mathcal{R}, r_{x} \in \mathscr{R}$ is the function given by $r_{x}\left(x_{1}, x_{2}, \ldots\right)=r\left(x, x_{1}, x_{2}, \ldots\right)$.

DEFINITION 3.7. Fix $r \in \mathcal{R}$ with $r[1] \equiv j$. Then $V\left(X_{r[2]}, \ldots, X_{r[k]} \mid X_{j}\right)=$ ess $\sup \left\{E\left(X_{r[t]} \mid X_{j}\right): t \in \mathcal{T}_{k}, t \geqslant 2\right\}$.

(For a definition and properties of "ess sup", see $[2, \S 1.6]$.)

Lemma 3.8. For $r \in \Re_{k}$, suppose $r[1] \equiv 1$ and let $P_{X_{1}}$ be the distribution of $X_{1}$. Then

$$
V\left(X_{r[2]}, \ldots, X_{r[k]} \mid X_{1}=x\right)=V\left(X_{r_{x}[2]}, \ldots, X_{r_{x}[k]}\right) \text { a.s. } P_{X_{1}} \text {. }
$$

Proof. Since $r_{x}[i] \neq 1$ for all $i>1$, the independence of $X_{1}, X_{2}, \ldots$ implies $X_{1}$ is independent of the whole sequence $X_{r_{x}[2]}, \ldots, X_{r_{x}[k]}$ for each $k \geqslant 2$ and each $x \in \mathbf{R}$ (although $X_{1}, X_{r[2]}, \ldots, X_{r[k]}$ in general are dependent, as can be seen in Example 3.4). Thus by the definitions of $X_{r}$ and $r_{x}, V\left(X_{r[2]}, \ldots, X_{r[k]} \mid X_{1}=x\right)=$ $V\left(X_{r_{x}[2]}, \ldots, X_{r_{x}[k]} \mid X_{1}=x\right)=V\left(X_{r_{x}[2]}, \ldots, X_{r_{x}[k]}\right)$ a.s. $P_{X_{1}}$.

The following example shows that the conclusion of the last lemma may fail without the assumption of independence, even for constant permutations of finite sequences.

EXAMPLE 3.9. Let $Y_{1}$ take only values 0 and 2, each with probability $\frac{1}{2}$, let $Y_{2} \equiv 1$, and let $Y_{3}=Y_{1}$. Then for the identity permutation $\pi \in \mathscr{P}_{3}$ (i.e. $\pi[i]=i, i=1,2,3$ ), clearly $\pi_{x}=\pi$ for all real $x$, and $V\left(Y_{\pi[2]}, Y_{\pi[3]} \mid Y_{1}=2\right)=V\left(Y_{2}, Y_{3} \mid Y_{1}=2\right)=2$, but $V\left(Y_{2}, Y_{3}\right)=1$.

DEFINITION 3.10. The worth of the collection of random variables $\left\{X_{1}, X_{2}, \ldots\right\}$, $W\left(\left\{X_{1}, X_{2}, \ldots\right\}\right)$, is given by $W\left(\left\{X_{1}, X_{2}, \ldots\right\}\right)=\sup \left\{V\left(X_{r}\right): r \in \Re\right\}$.

Thus the worth of a sequence is the most a player can expect to gain if he is free to select the order of observation of the random variables depending on past outcomes, and free to stop whenever he pleases.

The next theorem, the main result of this section, says that a player free to select the order of observation in an optimal stopping problem with independent random variables may do just as well using order selections which do not depend on past observations, as with ones which do.

Theorem 3.11. $W\left(\left\{X_{1}, X_{2}, \ldots\right\}\right)=\sup \left\{V\left(X_{\pi}\right): \pi \in \mathscr{P}\right\}$.

Proof. Clearly it is enough to show that for all $n \in \mathbf{N}$,

$$
W\left(\left\{X_{1}, \ldots, X_{n}\right\}\right)=\max \left\{V\left(X_{\pi}\right): \pi \in \mathscr{P}_{n}\right\} .
$$

The proof of (6) will use both forward and backward induction; forward induction on $n$ and backward induction within steps for fixed $n$. Clearly (6) is true for $n=1$ (and even $n=2$, since $\mathscr{P}_{2}=\mathscr{R}_{2}$ ). Assume it is true for $k=1, \ldots, n-1$, and fix $r \in \mathcal{R}_{n}$. Without loss of generality (otherwise reindex the $\left\{X_{j}\right\}$ ) it may be assumed that

$$
\begin{gathered}
r[1] \equiv 1 \text { and } \\
V\left(X_{2}, X_{3}, \ldots, X_{n}\right)=\max \left\{V\left(X_{\pi[2]}, \ldots, X_{\pi[n]}\right): \pi \in \mathscr{P}_{n}, \pi[1]=1\right\} .
\end{gathered}
$$

Since $\mathscr{P}_{n} \subset \mathcal{R}_{n}$, to prove (6) it is enough to show that

$$
V\left(X_{r}\right) \leqslant V\left(X_{1}, \ldots, X_{n}\right) \text {. }
$$


By a routine application of backward induction (e.g. [2, p. 50]), it follows that

$$
V\left(X_{r}\right)=\int_{A} x d P_{1}(x)+\int_{A^{c}} V\left(X_{r[2]}, \ldots, X_{r[n]} \mid X_{1}=x\right) d P_{1}(x),
$$

where $P_{1}$ is the distribution of $X_{1}$, and

$$
A=\left\{x \in \mathbf{R}: x>V\left(X_{r[2]}, \ldots, X_{r[n]} \mid X_{1}=x\right)\right\} .
$$

Now calculate

$$
\begin{aligned}
V\left(X_{r}\right) & =\int_{A} x d P_{1}(x)+\int_{A^{c}} V\left(X_{r_{x}[2]}, \ldots, X_{r_{x}[n]}\right) d P_{1}(x) \\
& \leqslant \int_{A} x d P_{1}(x)+\int_{A^{c}} V\left(X_{2}, \ldots, X_{n}\right) d P_{1}(x) \\
& \leqslant \int_{\left\{x>V\left(X_{2}, \ldots, X_{n}\right)\right\}} x d P_{1}(x)+\int_{\left\{x \leqslant V\left(X_{2}, \ldots, X_{n}\right)\right\}} V\left(X_{2}, \ldots, X_{n}\right) d P_{1}(x) \\
& =V\left(X_{1}, \ldots, X_{n}\right),
\end{aligned}
$$

where the first equality in (10) follows from (9) and Lemma 3.8; the first inequality from (7) and the induction hypothesis; the second inequality since (by backward induction again) it is optimal to stop at time 1 if and only if $X_{1}>V\left(X_{2}, \ldots, X_{n}\right)$; and the last equality by Lemma 3.5 . This completes the proof.

As the next example illustrates, the conclusion of Theorem 3.11 may fail without the assumption of independence, even for three random variables.

EXAmPLE 3.12. Define $Y_{1}, Y_{2}, Y_{3}$ jointly by $\left(Y_{1}, Y_{2}, Y_{3}\right)=(0,3,2),(0,3,4),(1,2,3)$, and $(1,4,3)$ each with probability $\frac{1}{4}$. It is easy to see that $V\left(Y_{\pi}\right)=13 / 4$ for all constant permutations $\pi \in \mathscr{P}_{3}$, but for any random permutation $r \in \mathcal{R}_{3}$ with $r[1] \equiv 1, r\left(0, x_{2}, x_{3}\right)[2]=3$, and $r\left(1, x_{2}, x_{3}\right)[2]=2$, that $V\left(X_{r}\right)=14 / 4 \leqslant$ $W\left(\left\{Y_{1}, Y_{2}, Y_{3}\right\}\right)$.

4. The advantage of order selection. Let $u, v, S$, and $W$ be as in $\S \S 2$ and 3 , and let $w=W\left(\left\{X_{1}, X_{2}, \ldots\right\}\right)$.

THEOREM 4.1. The set of all possible values of the ordered pairs $(v, w)$ for independent random variables uniformly bounded in $[0,1]$ is precisely the closed convex set $S$.

Proof. Since $W\left(\left\{X_{1}, X_{2}, \ldots\right\}\right)$ is clearly bounded below by $v=V\left(X_{1}, X_{2}, \ldots\right)$, and bounded above by $E\left(\sup _{n} X_{n}\right)$, it follows from Theorem 2.3 that $v \leqslant w \leqslant 2 v-$ $v^{2}$.

To see that every point in $S$ is a possible pair $(v, w)$ for some $X_{1}, X_{2}, \ldots$, fix $X_{1}$, $X_{2}, \ldots$ as in the proof of Theorem 2.3, and note that $W\left(\left\{X_{1}, X_{2}, \ldots\right\}\right)=$ $V\left(X_{2}, X_{1}, X_{3}, X_{4}, \ldots\right)=E\left(\sup _{n} X_{n}\right)$.

The corollaries analogous to (1) and (2) are now immediate.

COROLLARY 4.2. If $X_{1}, X_{2}, \ldots$ are independent nonnegative random variables, then

$$
W\left(\left\{X_{1}, X_{2}, \ldots\right\}\right) \leqslant 2 \sup \left\{E X_{t}: t \in \mathcal{T}\right\} .
$$


Corollary 4.3. If $X_{1}, X_{2}, \ldots$ are independent random variables uniformly bounded in $[a, a+b]$, then

$$
W\left(\left\{X_{1}, X_{2}, \ldots\right\}\right)-\sup \left\{E X_{t}: t \in \mathcal{T}\right\} \leqslant b / 4 .
$$

Moreover, it is clear that the bounds " 2 " in (11) and " $b / 4$ " in (12) are best possible.

As in $[3,4,5,7$, and 8$]$, probabilistic interpretations may be given to the results (11) and (12): (11) says that in an optimal stopping problem with independent nonnegative random variables, a player should never pay more than $v$ ( $=\sup \left\{E X_{t}: t \in \mathcal{T}\right\}$ ) for the privilege of rearranging the order of observation, even if allowed to do so sequentially depending on previous outcomes; and (12) says that in the uniformly bounded case, he should never pay more than $\frac{1}{4}$ for the privilege of rearranging the order of observation.

The next example, a slight variation of an example in the proof of [3, Proposition 1], shows that (11) may fail without the assumption of independence, even if the sequence of random variables is both Markov and a martingale.

EXAMPLE 4.4. Fix $\varepsilon>0$ and define random variables $Y_{1}, Y_{2}, \ldots, Y_{n}$ jointly by $P\left[\left(Y_{1}, \ldots, Y_{n}\right)=\left(1,1 / \varepsilon, \ldots, 1 / \varepsilon^{j}, 0, \ldots, 0\right)\right]=\varepsilon^{j}-\varepsilon^{j+1}$ if $0 \leqslant j \leqslant n-2$, and $=\varepsilon^{n-1}$ if $j=n-1$. Let $Y_{j} \equiv Y_{n}$ for $j>n$. Then $Y_{1}, Y_{2}, \ldots$ is Markov and a martingale, so $\sup \left\{E Y_{t}: t \in \mathcal{T}\right\}=E Y_{1}=1$, but

$$
\begin{aligned}
W\left(\left\{Y_{1}, Y_{2}, \ldots\right\}\right) & =V\left(Y_{n}, Y_{n-1}, \ldots, Y_{1}, Y_{n+1}, Y_{n+2}, \ldots\right) \\
& =E\left(\sup _{n} Y_{n}\right)=(n-1)(1-\varepsilon)+1 \nearrow n \quad \text { as } \varepsilon \searrow 0 .
\end{aligned}
$$

In fact, the above example is sharp in a sense, as is seen in the next result which follows easily from [3, Proposition 1].

Proposition 4.5. If $Y_{1}, Y_{2}, \ldots, Y_{n}$ are nonnegative random variables, then

$$
W\left(\left\{Y_{1}, \ldots, Y_{n}\right\}\right) \leqslant n\left\{\sup E Y_{t}: t \in \mathcal{T}_{n}\right\} .
$$

Similarly, (12) may fail without independence, even if the sequence is again both Markov and a martingale, as is seen easily from [3, Example 4.2].

If the random variables are uniformly bounded in $[a, a+b]$, the set $S$ in Theorem 4.1 must again be replaced by the set $S^{\prime}$ in (3).

Acknowledgement. The author is deeply indebted to Professor Lester Dubins, whose questions following a seminar in Berkeley led to the complete solutions of the prophet inequalities found in $\S 2$.

\section{BIBLIOGRAPHY}

1. A. Brunel and U. Krengel, Parier avec un prophète dans le cas d' un processus sous-additif, C. R. Acad. Sci. Paris 288 (1979), 57-60.

2. Y. S. Chow, H. Robbins and D. Siegmund, Great expectations: the theory of optimal stopping, Houghton Mifflin, Boston, Mass., 1971.

3. T. P. Hill and R. P. Kertz, Ratio comparisons of supremum and stop rule expectations, Z. Wahrsch. Verw. Gebiete 56 (1981), 283-285.

4. __ Additive comparisons of stop rule and supremum expectations of uniformly bounded independent random variables, Proc. Amer. Math. Soc. 83 (1981), 582-585.

5. Comparisons of stop rule and supremum expectations of i.i.d. random variables, Ann. Probab. 10 (1982), 336-345. 
6. T. P. Hill and V. C. Pestien, The advantage of using non-measurable stop rules, Ann. Probab. (to appear).

7. U. Krengel and L. Sucheston, Semiamarts and finite values, Bull. Amer. Math. Soc. 83 (1977), 745-747.

8. On semiamarts, amarts, and processes with finite value, Adv. Appl. Probability 4 (1978), $197-266$.

School of Mathematics, Georgia Institute of Technology, Atlanta, Georgia 30332

Current address: Department of Mathematics, University of Leiden, Wassenaarseweg 80, Postbus 9512, 2300 RA Leiden, The Netherlands 